\title{
Low-thickness high-quality aluminum nitride films for super high frequency solidly mounted resonators
}

\author{
E. Iborra , M. Clement ，J. Capilla ，J. Olivares ，V. Felmetsger
}

\section{A R T I C L E I N F O}

\section{Article history:}

Received 6 July 2011

Received in revised form 20 October 2011

Accepted 3 November 2011

Available online 11 November 2011

\section{Keywords:}

Piezoelectric AlN

Crystal quality

$\mathrm{AC}$ reactive sputtering

Two-step deposition

High frequency solidly mounted resonator

Electromechanical coupling factor

Quality factor

\begin{abstract}
A B S T R A C T
We investigate the sputter growth of very thin aluminum nitride (AIN) films on iridium electrodes for electroacoustic devices operating in the super high frequency range. Superior crystal quality and low stress films with thicknesses as low as $160 \mathrm{~nm}$ are achieved after a radio frequency plasma treatment of the iridium electrode followed by a two-step alternating current reactive magnetron sputtering of an aluminum target, which promotes better conditions for the nucleation of well textured AlN films in the very first stages of growth. Solidly mounted resonators tuned around $8 \mathrm{GHz}$ with effective electromechanical coupling factors of $5.8 \%$ and quality factors $Q$ up to 900 are achieved.
\end{abstract}

\section{Introduction}

Wireless communications systems have undergone an extraordinary evolution since the first mobile phones operating in $800 \mathrm{MHz}$ range appeared in the market in the early 70 s. Current mobile phones (GSM, WCDMA, DCS, etc.) operate in the ultra high frequency range at frequencies between $800 \mathrm{MHz}$ and $2200 \mathrm{MHz}$. The extremely rapid expansion of the mobile phone market is boosting the development of new communication systems addressing the super high frequency (SHF) range, to prevent radio wave congestion or interference and allow the efficient use of the frequency spectrum [1-3].

High frequency filters are fundamental components of wireless communications systems. Filters based on bulk acoustic wave (BAW) resonators have been extensively developed during the last ten years and have become the best choice for very high frequency filtering applications [4]. BAW filters are made up of two sets of BAW resonators tuned to two close resonant frequencies connected through different topologies. Each resonator has a simple structure made up of a piezoelectric film sandwiched between two electrodes (piezoelectric stack). Aluminum nitride (AIN) is the preferred active material owing to its good acoustic properties and its compatibility with silicon technologies [5]. The performance of the devices is improved by acoustically isolating the piezoelectric stack from the

\footnotetext{
* Corresponding author. Tel.: + 34 616950336; fax: + 343367216 . E-mail address: mclement@etsit.upm.es (M. Clement).
}

substrate either by an air cavity, which gives rise to the so-called film bulk acoustic resonators [6], or by placing an acoustic reflector composed of a set of alternated layers of high and low acoustic impedance among the piezoelectric stack and the substrate, in which case we obtain solidly mounted resonators (SMR) [7].

The resonant frequency $f_{\mathrm{r}}$ of an ideal BAW resonator (free standing piezoelectric stack with electrode thicknesses tending to zero) is set by fixing the thickness of the piezoelectric layer to $\lambda / 2$, although in practice the exact thickness of the piezoelectric slab depends on the weight of all surrounding layers. Current BAW filters operating around $2 \mathrm{GHz}$ require AIN films of around $1500 \mathrm{~nm}$-thick, which are routinely achieved with excellent piezoelectric properties through optimized industrial sputtering processes [8]. SHF applications require a remarkable reduction of the thickness of the AIN films to values for which their crystal quality and piezoelectric activity drop significantly [9], owing to the poor crystal quality of the material in the initial stages of growth. BAW resonators based on ultra thin AIN films (from $150 \mathrm{~nm}$ to $300 \mathrm{~nm}$ ) are being developed in some research institutions and companies [1-3,10-14]. However, the crystal quality of such films is still far from that of thicker films. This, along with the increase of the acoustic losses with frequency, explains why high frequency resonators exhibiting simultaneously great values of the effective electromechanical coupling coefficient $\left(k_{\text {eff }}^{2}\right)$ and quality factor $(Q)$ are hardly achieved, particularly in the case of SMRs $[11,12]$. So far, competitive high frequency resonators with $k_{\mathrm{eff}}^{2}$ greater than $6 \%$ have only been achieved in devices suspended over an air cavity [1-3]. 
In this work we investigate the growth of very thin AIN films on iridium (Ir) electrodes by a special alternating current (AC) reactive sputtering process that controls the interaction between the energetic particles of the glow discharge with both the Al target and the substrate during the deposition process. The aim is to achieve films exhibiting good crystal quality from the very first stages of growth. Once the piezoelectric properties of the AIN films optimized, SMRs tuned to frequencies close to $8 \mathrm{GHz}$ are designed through a careful iterative process that takes into account the nature of the electrodes, the ratio of the thickness of the electrodes to that of the piezoelectric layer, and the acoustic isolation of the reflector; the goal is to achieve SMR devices operating at SHF and exhibiting simultaneously large effective electromechanical coupling factor $\left(k_{\mathrm{eff}}^{2}\right)$ and quality factor $(Q)$.

\section{Experimental techniques}

AIN films were deposited in an Endeavor-AT physical vapor deposition cluster tool of OEMGroup Inc. One of the process modules is equipped with an S-Gun magnetron for alternating current $(A C)$ $(40 \mathrm{kHz})$ reactive sputtering with capability for independent control of the film crystal orientation, stress, and uniformity [15]. The deposition rate was $48 \mathrm{~nm} / \mathrm{min}$ in all experiments. Prior to AlN deposition, the substrate surface was treated by low energy $\mathrm{Ar}$ ions either in a separate planarized $\mathrm{RF}(13.56 \mathrm{MHz}$ ) (PRF) etch module producing a capacitively coupled plasma, or in the deposition module (in-situ etch). Deposition processes were performed either without external heating $\left(\mathrm{T}<300{ }^{\circ} \mathrm{C}\right)$ or at elevated temperatures (up to $500{ }^{\circ} \mathrm{C}$ ) using an external infrared heater to warm the wafer before or during deposition.

The crystal structure of the AIN and Ir films was assessed by scanning electron microscopy (SEM) and X-ray diffraction (XRD). XRD measurements were performed with a Supratech XPERT-PRO diffractometer by recording the $\theta / 2 \theta$ patterns between $16^{\circ}(\theta)$ and $40^{\circ}(\theta)$ using the $\mathrm{K} \alpha 1,2$ doublet of a $\beta$-filtered $\mathrm{Cu}$ anode. Rocking curves (RC) around the most intense reflections, i.e. the AIN 00.2 at $18.02^{\circ}(\theta)$ and the Ir 111 at $20.32^{\circ}(\theta)$, were measured as well and fitted by Gaussian curves to deduce their full width at half maximum (FWHM) [16]. Infrared reflectance measurements between $200 \mathrm{~cm}^{-1}$ and $4000 \mathrm{~cm}^{-1}$ with unpolarized light at an angle of incidence of $30^{\circ}$ were used to assess the texture of the AIN films [17] and to obtain stress values independent of the substrate curvature. The polar orientation of AIN films was investigated by measuring their etch-ability in hot phosphoric acid $[18,19]$.

SMRs were fabricated by building Ir/AIN/Ir stacks on top of acoustic reflectors formed by five alternated layers of $\mathrm{SiO}_{2}$ and Ir. The combination of the high acoustic impedance Ir layer $\left(120 \times 10^{6} \mathrm{~kg} / \mathrm{m}^{2} \cdot \mathrm{s}\right)$ [20] with a specifically developed porous- $\mathrm{SiO}_{2}\left(\mathrm{p}-\mathrm{SiO}_{2}\right)$ of low acoustic impedance $\left(9 \times 10^{6} \mathrm{~kg} / \mathrm{m}^{2} \cdot \mathrm{s}\right)$ [21] provided the desired acoustic isolation. The $\mathrm{p}-\mathrm{SiO}_{2}$ uppermost layer of the mirror was mechanically polished to reduce roughness to less than $1 \mathrm{~nm}$. Ti/Mo or Ti/W seed layer was used to promote the growth of highly (111)-oriented Ir layers with narrow RC (FWHM in the range of $2.5^{\circ}$ ). Ir bottom electrodes were then deposited either by e-beam evaporation at a substrate temperature of $420^{\circ} \mathrm{C}$ or by pulsed DC sputtering at $350{ }^{\circ} \mathrm{C}$. Both methods provided (111)-oriented Ir underlayers that offered a compatible surface for obtaining highly c-axis textured AIN films [22] and provided, additionally, the high acoustic impedance required for the good operation of BAW resonators.

The piezoelectric activity of AIN films and the performance of the devices were assessed by measuring the electrical reflection coefficient $\left(S_{11}\right)$ at frequencies ranging from $30 \mathrm{kHz}$ to $10 \mathrm{GHz}$, using an Agilent PNA N5230A network analyzer. The electrical impedance of the resonators, derived from $S_{11}$, was fitted with Mason's model [23] to obtain accurate values of both material and effective electromechanical coupling factors ( $k_{\text {mat }}^{2}$ and $k_{\mathrm{eff}}^{2}$ ). The method allows assessing the AIN properties independently of the design of the device.
Quality factors at resonant $\left(Q_{x}\right)$ and antiresonant $\left(Q_{a}\right)$ frequencies were derived from the slope of the phase of the electrical impedance.

\section{Results and discussion}

\subsection{Setting of the deposition parameters}

The deposition process started with a thermal treatment of the Ir electrode under vacuum to desorb oxygen and water vapor; then, a soft bombardment with low-energy $\mathrm{Ar}$ ions prepared the metallic surface to promote the growth of AIN microcrystals with identical polar orientation to maximize the piezoelectric response of the film. The power of this pre-deposition bombardment had to be carefully tuned, as too low values ( $30 \mathrm{~W}$ ) yielded poorly textured AIN films (wide RCs), whereas too high values $(200 \mathrm{~W}$ ) produced AIN films with low etch-ability in hot phosphoric solutions, which has been associated to the formation of inverted domains $[24,25]$. Thus, the overall pre-treatment of the surface acted in two different ways; firstly by modifying the state of the Ir surface (removing contaminants) and secondly by homogenizing the activation state of the surface to promote the growth of AIN grains with a single polar orientation. Once the surface prepared, the growth conditions of ultra thin AIN films had to ensure not only a strong c-axis texture, but also a homogeneously distributed fine-grain microstructure at the very first stage of deposition. Indeed, wet etch experiments of 1 um-thick AIN films in hot phosphoric acid revealed that easily etched films display a fine grain structure in the early stages of film growth, which evolved into a columnar structure, whereas highly etch-resistive films (containing thus inversion domains) exhibited a columnar grain structure noticeably larger (grain width larger approximately by a factor of 2) that already developed on the early stages of growth [19]. To stimulate the growth of the fine-grain microstructure, the adatom mobility in these first stages had to be large to induce the nucleation. This was achieved by starting the deposition at a high substrate temperature $\left(400{ }^{\circ} \mathrm{C}\right.$ to $450^{\circ} \mathrm{C}$ ) and a high nitrogen flow ensuring that the S-gun magnetron target remained in the deep poisoned mode. If these sputter conditions were maintained, the basal diameter of the grains tended to increase to form compact films with wide grains and narrow RCs. However, this situation was not attained in ultra thin films for which the crystallinity is better if the grain diameter is kept in a low homogeneous value, which occurred if the substrate temperature was reduced during deposition. In summary, the films were deposited following a two-step process: the first $50 \mathrm{~nm}$ were deposited at high temperature $\left(400-450^{\circ} \mathrm{C}\right)$ and high nitrogen gas flow, which promoted the nucleation of small size grains preferably oriented by nitrogen basal plane on top, as suggested by wet etch experiments [19]; the remaining film was deposited at a wafer temperature of about $300-350{ }^{\circ} \mathrm{C}$ without external heating, with the $\mathrm{N}_{2}$ flux reduced to the value enabling the target to remain in a poison mode, but at a work point located closer to the transition zone between the poison and the metallic mode on the hysteresis curve [15]. SEM images of the surface and cross section of a typical $200 \mathrm{~nm}$-thick AIN film grown using a two-step process are presented in Fig. 1. The two pictures show that the film is formed by a dense columnar structure of grains with a basal diameter of around $40 \mathrm{~nm}$; the transition between the first and second step is clearly visible in the cross section image. The arrow marks the transition between the first and second deposition steps.

AlN films tended to exhibit residual stress that could be adjusted by controlling the influx of energetic particles from the plasma to the substrate during growth. This was commonly achieved by directly biasing the substrate or by varying the pressure in the chamber, which also affected other properties of the films. In order to control stress independently of other film and process parameters, the S-gun was polarized with respect to the chamber walls by means of a variable resistance, which allowed the reconfiguration of the 

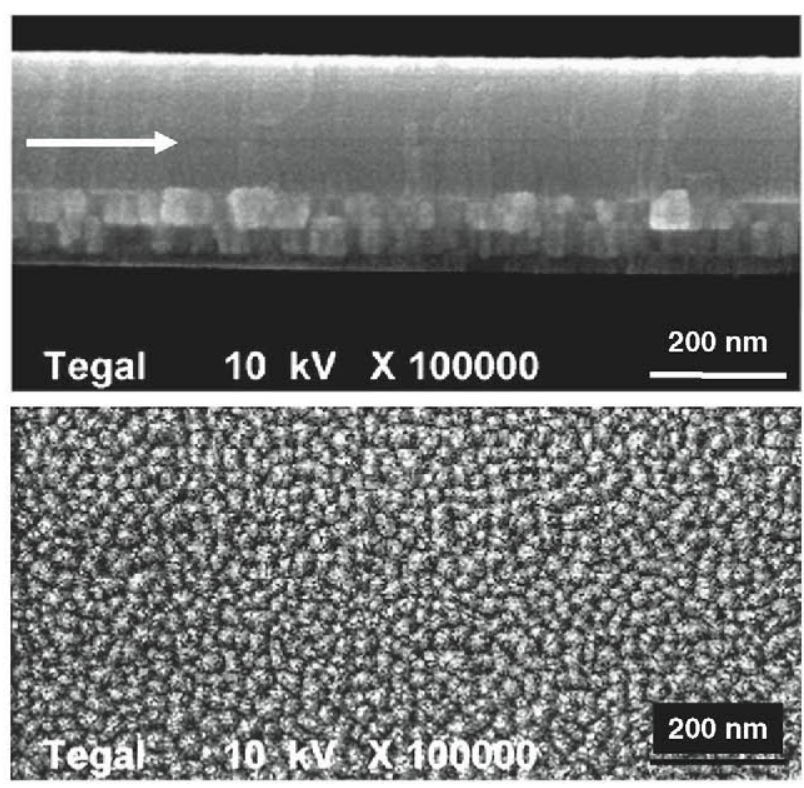

Fig. 1. Cross-section and plane-view SEM images of a typical $200 \mathrm{~nm}$-thick AlN film. The arrow marks the transition between the first and second deposition steps.

electric field lines inside the chamber, enabling electrons and ions to be fed to the walls before reaching the substrate [26]; the influx of energetic particles was therefore controlled independently of pressure. The stress of AIN on Ir could be set from strongly tensile $(+1000 \mathrm{MPa})$ to strongly compressive $(-1000 \mathrm{MPa})$. Most of the films deposited for this study had a reproducible tensile stress lower than $200 \mathrm{MPa}$.

An important consideration to control the crystal quality of the piezoelectric layer was the smoothness of the underlying substrate. Fig. 2 depicts the width of the RCs around the AIN $(00 \cdot 2)$ reflection and the value of $k^{2}$ mat as a function of the crystal quality of the Ir bottom electrode. It can be noticed that AIN films grown on polished substrates exhibit excellent piezoelectric activity, almost independent of the RC of the underlying Ir electrode (ranging from $2^{\circ}$ to $5^{\circ}$ ) and the $\mathrm{RC}$ around the $(00 \cdot 2)$-AIN reflection (ranging from $1.5^{\circ}$ to $3^{\circ}$ ). The widening of the RCs of both Ir and AIN is very likely related with a "geometrical" effect (growth on tilted surfaces) rather than to a real worsening of the crystalline structure [27].

Hence, it was found that it is possible to produce highly oriented AIN films even on Ir electrodes of different quality. The best achievements were AIN films $200 \mathrm{~nm}$-thick with a RC $1.57^{\circ}$-wide grown on Ir substrates with a RC of $3.8^{\circ}$. The crystal quality of such films is almost

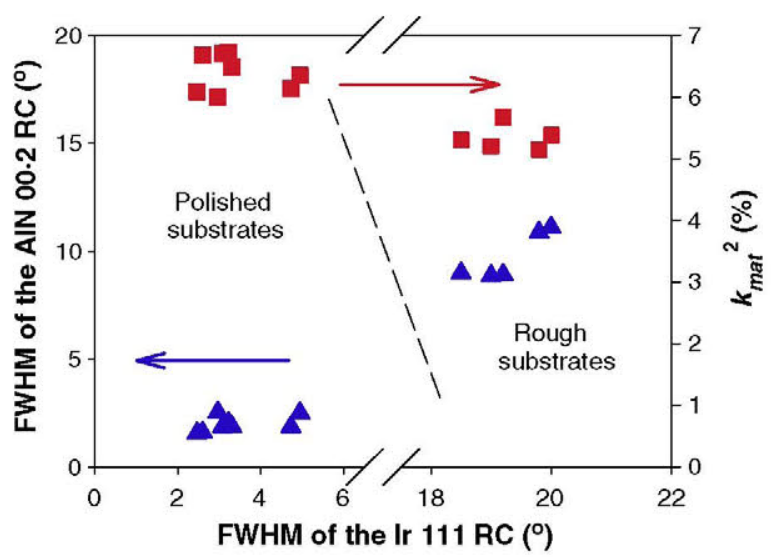

Fig. 2. FWHM of the RC around the $(00 \cdot 2)$ AIN XRD peak ( $\mathbf{\Delta})$ and $k_{\text {mat }}^{2}(\boldsymbol{\nabla})$ as a function of the FWHM of the RC around the (111) Ir peak. as good as that obtained in $1 \mu \mathrm{m}$-thick AIN films deposited under the same deposition conditions and identical substrates, as the XRD patterns and RCs shown in Fig. 3 demonstrate. In summary, the best performing AIN films were achieved on polished substrates under the following sputter conditions: in-situ wafer annealing in vacuum, low-power RF plasma etch in the PRF module, and 2-step AIN deposition.

\subsection{High frequency resonators}

The viability of the AIN films for high frequency filter applications was demonstrated through the performance of the SMRs tuned at $8 \mathrm{GHz}$. The acoustic reflectors made of five alternated layers of p$\mathrm{SiO}_{2}$ and Ir were designed to obtain an acoustic transmittance of the longitudinal resonant mode lower than $-35 \mathrm{~dB}$ in a wide frequency band that included the resonant frequency and, simultaneously, an acoustic transmittance of the shear modes lower than $-20 \mathrm{~dB}$. This was achieved by setting the thickness of the layers in the mirror to values different from $\lambda / 4$, according to [28]. This design improves the acoustic isolation at the resonant frequency, which increases the quality factor of the SMR compared to that obtained on conventional symmetric $\lambda / 4$ Bragg mirrors. Once the acoustic reflector is designed and tuned to the desired resonance frequency, a simulation of the frequency response of the SMRs was performed in order to obtain the thickness of the layers composing the piezoelectric stack (AIN and Ir electrodes) that would maximize the values of $k_{\text {eff }}^{2}$ and $Q_{\text {a }}$. To simplify the problem the top and bottom electrodes were taken of identical thickness. Fig. 4 shows values of AIN thickness, $k_{\text {eff }}^{2}$ and $Q_{a}$ derived from the simulations performed by varying the electrode thickness. Although all the piezoelectric stacks shown in Fig. 4 produce a resonance at $8 \mathrm{GHz}$, the maximum value of $k_{\mathrm{eff}}^{2}$ is achieved for a unique combination of thicknesses, which corresponds to $260 \mathrm{~nm}$-thick AIN layer sandwiched between two $45 \mathrm{~nm}$-thick Ir electrodes.

One of the main problems of high frequency resonators is the use of very thin electrodes, which produces a severe increase of the series resistance and a significant reduction of the quality factor at the resonant frequency $Q_{r}$. The situation gets worse when using high density metals like Ir, which imposes a severe reduction of the thickness of the layer in order to keep the resonant frequency constant. On the other hand, $Q_{a}$ mainly depends on the acoustic losses through the materials of the whole structure. To achieve the best design a compromise has to be reached between the values of $k_{\text {eff }}^{2}$ and $Q$. Therefore, we have considered that $75 \mathrm{~nm}$-thick Ir electrodes, which yield series resistances around $1 \Omega$ and $Q_{r}$ of 150 are acceptable values.

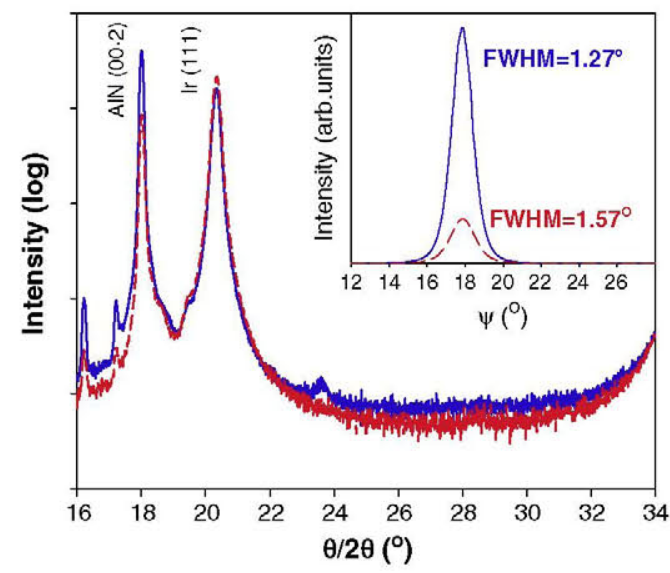

Fig. 3. X-ray diffraction $\theta / 2 \theta$ patterns of $1 \mu \mathrm{m}$ (solid blue) and $200 \mathrm{~nm}$-thick (dashed red) AlN films grown on Ir electrodes deposited over polished Bragg mirrors. Inset shows their rocking curves around the $(00 \cdot 2)$-AlN reflection. 


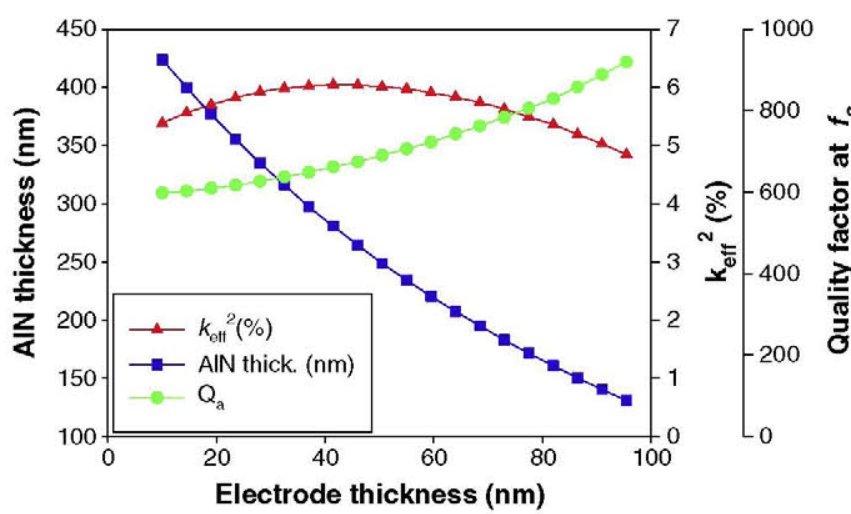

Fig. 4. Simulated AlN thickness $(\boldsymbol{\nabla}), k_{\mathrm{eff}}^{2}(\mathbf{\Delta})$, and $\mathrm{Q}_{a}(\boldsymbol{0})$ as a function of the thickness of the Ir electrodes for resonators tuned at $8 \mathrm{GHz}$ and AlN layers with $k_{\text {mat }}^{2}$ of $6.5 \%$. Acoustic losses in AIN are considered.

Fig. 5 shows the frequency measurements of a typical SMR tuned at $8 \mathrm{GHz}$; for this device we obtained $k_{\mathrm{eff}}^{2}=5.8 \%, \mathrm{Q}_{\mathrm{r}}=160$ and $Q_{\mathrm{a}}=930$. The $k_{\text {mat }}^{2}$ required to fit the frequency response of this device with Mason's model was 6.7\%. A summary of the characteristics of the resonators is shown in Table 1 , where the results achieved by other authors in both FBAR and SMR devices are also included for comparison. These results show that although AIN films are of very good quality, the design of the piezoelectric stack can be further improved. A reduction of the series resistance is mandatory, which could be achieved by using a metallic layer of a low density material, such as $\mathrm{Al}$ or $\mathrm{Ti}$, under the Ir bottom electrode. This could increase the value of $k_{\text {eff }}^{2}$ while keeping good values of $Q_{r}$.

\section{Conclusions}

In summary, a careful adjustment of the parameters of an AC magnetron reactive sputtering process enables growing less-than-200$\mathrm{nm}$ thick AIN films on Ir substrates whose structural and piezoelectric properties are almost as good as the best obtained for $1 \mu \mathrm{m}$-thick films. This material allows one to achieve SMRs tuned at $8 \mathrm{GHz}$ with $k_{\mathrm{eff}}^{2}$ of $5.8 \%$ and $Q_{\mathrm{a}}$ of 930 ; their performance at high frequency can be further improved by optimizing the electrode design.

\section{Acknowledgment}

This work has been supported by Ministerio de Educación y Ciencia of Spain through project MAT2010-18933, Comunidad de Madrid and UPM through the IV PRICIT Programme, and through the European Regional Development Fund (FEDER).

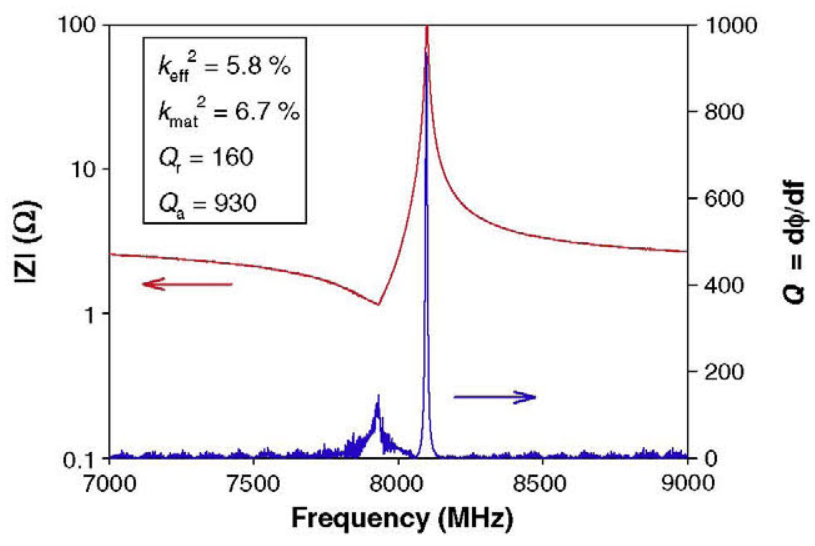

Fig. 5. Modulus of the impedance $\mathrm{Z}(\mathrm{Z}=\mathrm{Z} \angle \Phi)$ and quality factor $\mathrm{Q}(\mathrm{Q}=\mathrm{d} \phi / \mathrm{df})$ as a function of frequency of a SMR tuned at $8 \mathrm{GHz}$ and containing a $200 \mathrm{~nm}$-thick AlN layer.
Table 1

Characteristics of high frequency AIN-based SMRs and FBARs.

\begin{tabular}{|c|c|c|c|c|c|c|c|c|}
\hline \multirow{2}{*}{$\frac{\text { Device }}{\text { Reference }}$} & \multicolumn{5}{|l|}{ FBAR } & \multicolumn{3}{|l|}{ SMR } \\
\hline & {$[1,13]$} & & & {$[3,14]$} & & [11] & [12] & Iborra \\
\hline Electrode & $\mathrm{Ru}$ & & & $\mathrm{Ti} / \mathrm{Al}$ & & Pt & Pt & Ir \\
\hline FWHM-RC $\left({ }^{\circ}\right)$ & & & & 2 & 2.3 & 3.5 & 1.8 & 1.57 \\
\hline $\mathrm{f}(\mathrm{GHz})$ & 9 & 20 & 24.8 & 10 & 20 & 9.2 & 8 & 8 \\
\hline$k_{\text {eff }}^{2}(\%)^{\mathrm{a}}$ & 6.0 & 6.25 & 6.01 & 6.30 & 6.1 & -4 & 5.9 & 5.8 \\
\hline $\mathrm{Qr}^{\mathrm{a}}$ & 479 & 189 & 285 & 330 & 280 & 282 & 362 & 930 \\
\hline $\mathrm{Ca}^{\mathrm{a}}$ & 314 & 171 & 291 & & & & & 160 \\
\hline
\end{tabular}

a Note: The values of $k^{2}$ eff and $Q$ published by the different authors are not necessarily obtained using identical definitions.

\section{References}

[1] M. Hara, T. Yokoyama, T. Sakashita, S. Taniguchi, M. Iwaki, T. Nishihara, M. Ueda, Y. Satoh, Jpn. J. Appl. Phys. 49 (2010) 07HD13.

[2] S. Tanifuji, Y. Aota, S. Kameda, T. Takagi, K. Tsubouchi, Rome, Italy, September 20 23, 2009, IEEE International Ultrasonics Symposim proceedings, , 2009, p. 2170.

[3] Y. Yoshino, J. Appl. Phys. 105 (2009) 061623.

[4] L. Mourot, P. Bar, G. Parat, P. Ancey, S. Bila, J.F. Carpentier, IEEE Microw. Mag. 9 (5) (2008) 104

[5] A. Ababneh, U. Schmid, J. Hernando, J.L. Sánchez-Rojas, H. Seidel, Mater. Sci. Eng. B 172 (2010) 253.

[6] L. Callaghan, V. Lughi, N. MacDonald, D. Clarke, IEEE Trans. Ultrason. Ferroelectr. Freq. Control 53 (2006) 1001.

[7] E. Schmidhammer, H. Heinze, M. Schmiedgen, M. Mayer, A. Link, Vancouver, Canada, October 36,2006 , IEEE International Ultrasonics Symposium proceedings, , 2006, p. 329.

[8] R. Lanz, C. Lambert1, L. Senn, L. Gabathuler, G. Reynolds, Vancouver, Canada, October 3 6, 2006, IEEE International Ultrasonics Symposium proceedings (2006) 1481.

[9] F. Martin, P. Muralt, M.A. Dubois, A. Pezous, J. Vac. Sci. Technol. A 22 (2004) 361.

[10] V. Felmetsger, P. Laptev, R. Graham, J. Vac. Sci. Technol. A 29 (2011) 21014.

[11] H.P. Loebl, M. Klee, C. Metzmacher, W. Brand, R. Milsom, P. Lok, Mater. Chem. Phys. 79 (2003) 143.

[12] R. Lanz, P. Muralt, IEEE Trans. Ultrason. Ferroelectr. Freq. Control 52 (6) (2005) 936.

[13] T. Yokoyama, M. Hara, M. Ueda, Y. Satoh, Biejing, November 2-5, 2008, IEEE International Ultrasonics Symposim proceedings, , 2008, p. 598.

[14] K. Umeda, H. Kawamura, M. Takeuchi, Y. Yoshino, Vacuum 83 (2009) 672.

[15] V.V. Felmetsger, P.N. Laptev, S.M. Tanner, Surf. Coat. Technol. 204 (2009) 840.

[16] J. Olivares, S. González-Castilla, M. Clement, A. Sanz-Hervás, L. Vergara, J. Sangrador, E. Iborra, Diamond Relat. Mater. 16 (2007) 1421.

[17] E. Iborra, M. Clement, L. Vergara, A. Sanz-Hervás, J. Olivares, J. Sangrador, Appl. Phys. Lett. 88 (2006) 231901.

[18] J. Olivares, S. González-Castilla, M. Clement, A. Sanz-Hervás, L. Vergara, J. Sangrador, E. Iborra, Diamond Relat. Mater. 16 (2007) 1421.

[19] S.M. Tanner, V.V. Felmetsger, J. Vac. Sci. Technol. A 28 (2010) 69.A

[20] A. Devos, J. Olivares, M. Clement, E. Iborra, S. González-Castilla, N. Rimmer, A. Rastogi, New York, USA, October 2831,2007 , IEEE International Ultrasonics Symposium proceedings, , 2007, p. 1433.

[21] J. Olivares, E. Wegmann, J. Capilla, E. Iborra, M. Clement, L. Vergara, R. Aigner, IEEE Trans. Ultrason. Ferroelectr. Freq. Control 57 (1) (2010) 23.

[22] M. Clement, J. Olivares, E. Iborra, S. González-Castilla, N. Rimmer, A. Rastogi, Thin Solid Films 517 (2009) 4673.

[23] J.F. Rosenbaum, Bulk Acoustic Wave Theory and Devices, Artech House, Boston, London, 1988.

[24] T. Kamohara, M. Akiyama, N. Kuwano, Appl. Phys. Lett. 92 (2008) 093506.

[25] J.A. Ruffner, P.G. Clem, B.A. Tuttle, D. Dimos, D.M. Gonzales, Thin Solid Films 354 (1999) 256.

[26] V.V. Felmetsger, P.N. Laptev, S.M. Tanner, J. Vac. Sci. Technol. A 27 (2009) 417.

[27] S. Mishin, D.R. Marx, B. Sylvia, V. Lughi, K.I. Turner, D.R. Clarke, Honolulu, USA, October 58,2003 , IEEE International Ultrasonics Symposium proceedings (2003) 2028.

[28] S. Marksteiner, J. Kaitila, G.G. Fattinger, R. Aigner, Rotterdam, The Netherlads, September 19 21, 2005, IEEE International Ultrasonics Symposium proceedings, 2005, p. 329. 